\title{
A model experiment to understand the oral phase of swallowing of Newtonian liquids.
}

\author{
P.Hayoun ${ }^{\mathrm{a}}$, J.Engmann ${ }^{\mathrm{a}}$, S.Mowlavi $^{\mathrm{a}}$, B.Le Reverend $^{\mathrm{a}}$, A.Burbidge ${ }^{\mathrm{a}}$, \\ M.Ramaioli ${ }^{\mathrm{a}, \mathrm{b}, *}$ \\ ${ }^{a}$ Nestlé Research Center, Route du Jorat 57, 1000 Lausanne 26, Switzerland \\ ${ }^{b}$ Department of Chemical and Process Engineering, Faculty of Engineering and Physical \\ Sciences, University of Surrey, Guildford, GU2 7XH, United Kingdom
}

\begin{abstract}
A model experiment to understand the oral phase of swallowing is presented and used to explain some of the mechanisms controlling the swallowing of Newtonian liquids. The extent to which the flow is slowed down by increasing the viscosity of the liquid or the volume is quantitatively studied. The effect of the force used to swallow and of the gap between the palate and the roller used to represent the contracted tongue are also quantified. The residual mass of liquid left after the model swallow rises strongly when increasing the gap and is independent of bolus volume and applied force. An excessively high viscosity results in higher residues, besides succeeding in slowing down the bolus flow.

A realistic theory is developed and used to interpret the experimental observations, highlighting the existence of an initial transient regime, at constant acceleration, that can be followed by a steady viscous regime, at constant velocity. The effect of the liquid viscosity on the total oral transit time is lower when the constant acceleration regime dominates bolus flow. Our theory suggests also that tongue inertia is the cause of the higher pressure observed at the back of the tongue in previous studies.
\end{abstract}

The approach presented in this study paves the way toward a mechanical

\footnotetext{
${ }^{*}$ Corresponding author

Email address: m.ramaioli@surrey.ac.uk (M.Ramaioli)
}

Preprint submitted to Journal of Biomechanics

November 9, 2015 
model of human swallowing, that would facilitate the design of novel, physicallysound, dysphagia treatments and their preliminary screening before in vivo evaluations and clinical trials.

Keywords: Fluid mechanics, Oral cavity, Tongue, Swallowing, Bolus, Flow, Peristalsis, Palate, Viscosity.

2014 MSC: 00-01, 99-00

\section{Introduction}

Swallowing occurs about one thousand times per day to transport subconsciously saliva and to transport voluntarily food from the mouth to the stomach (Dodds, 1989). Swallowing disorders, also known as dysphagia, can be the 5 consequence of several pathologies, such as throat and neck cancer, stroke, dementia, or other neurodegenerative conditions. The higher prevalence among elderly subjects justifies increased attention in countries with aging populations. Dysphagia management is crucial for providing adequate nutrition and hydration while minimizing the risk of choking, aspiration and resulting pulmonary diseases.

Despite such importance, swallowing is still not fully understood, because it involves the complex, coordinated contraction of different muscles located in and around the tongue, larynx, pharynx and oesophagus. The bolus flow starts in the mouth under the pressure produced by the tongue surface, moving toward the hard palate. The pressure and the total work provided by the tongue during the squeezing action of different jelly-type foods was recently studied (Yokoyama et al., 2014) and maximum pressures were measured in the range $5-40 \mathrm{kPa}$. Other groups (Nicosia et al. 2000, Youmans and Stierwalt, 2006) reported previously similar figures and highlighted also that pressure varies along the tongue position and is higher at the back. The mean peak tongue pressure was also found to remain similar across different age groups, although maximum tongue strength was found to decrease with age (Youmans and Stierwalt, 
2006: Utanohara et al., 2008). Clavé et al. (2006) showed that brain damage or neurodegenerative conditions result in decreased tongue pressure, decreased bolus kinetic energy and increased transit time.

The volume of the bolus swallowed was shown to vary with the viscosity of the food (Adnerhill et al., 1989). For thin liquids, the volume ranges from $1 \mathrm{~mL}$ (saliva bolus) to $20 \mathrm{~mL}$ (cup drinking), and decreases when drinking hot fluids or sipping.

A simple theory, considering the transient motion of two rigid plates as a model for the tongue and the palate, has been proposed to describe the fluid mechanics of bolus ejection from the oral cavity by Nicosia and Robbins $(2001)$. This theory has highlighted that liquid density can potentially play an important role in swallowing. However, in that simple system, the tongue and the palate are separated by a spatially uniform gap that decreases with time and an infinite time is needed to complete the ejection of the bolus. In turn, it is extremely difficult to perform any direct comparison with in vivo data.

To bridge the gap between theory and in vivo experiments in the field of mastication, Woda et al. (2010) developed an artificial masticator and studied in a controlled way the effect of the different process parameters, while overcoming the difficulties linked to in vivo observations. Similarly, De Loubens et al. (2010) and De Loubens et al. (2011) proposed a simple pharyngeal peristalsis simulator and a numerical analysis to study the flow and the resulting coating thickness.

Numerical simulations were recently used to reproduce mastication and hu45 man swallowing, but the complexity of this free surface flow requires nonconventional algorithms such as SPH (Ho et al. (2014)) or MPS (Kikuchi et al. (2015)).

Mackley et al. (2013) characterized the rheology of dysphagia thickeners and proposed for the first time a qualitative model experiment replicating tongue 50 peristalsis. Our study proposes a quantitative model experiment to understand swallowing and in particular the effect of bolus physical properties on the bolus 
transit time and the residues left during the oral phase of swallowing.

\section{Materials and methods}

\subsection{Materials}

Aqueous glycerol solutions are used in this study because they allow a wide viscosity variation, while showing a simple Newtonian rheology. The liquid viscosity of the aqueous glycerol solutions was characterized using a rheometer Physica MCR-500 by Anton Paar, under simple shear with a concentric cylinder geometry. The water concentration was varied in the range $0,2,5,20,50 \% \mathrm{wt}$ ( 0 to $440 \mathrm{~g} / \mathrm{L}$ ), to obtain a viscosity range from $\mu=1.185 \mathrm{~Pa}$ s to $\mu=0.006 \mathrm{~Pa}$ $\mathrm{s}$ at $22^{\circ} \mathrm{C}$. A red dye powder was added for enhancing contrast in the images, with no impact on the rheological properties of the liquids.

\subsection{Methods}

To simulate the oral phase of human swallowing, we developed and optimized the mechanical model designed initially by Mackley et al. (2013). The model experiment used in this study is shown in Figure 1. The top rigid wall is a bidimensional approximation of a human palate, with a constant curvature along $\theta$.

This experimental setup simulates two main actions of the tongue during swallowing . A roller, attached to a pivoting arm, mimics the contracted part of the tongue, propelling the bolus. Its position during the experiment is defined by the angle $\theta$ (see Figure 1). A thin, compliant tubing made of a dialysis membrane mimics the ability of the tongue at rest to hold the fluid in mouth. When this is empty and flat, its width is $\mathrm{w}_{\mathrm{T}}=23 \mathrm{~mm}$ and its thickness is 0.1 $75 \mathrm{~mm}$, including the adhesive tape. We define $\mathrm{h}$ the gap between the roller and the palate and h' the initial gap between the roller and the tube, the difference being the membrane thickness. Before each experiment, the soft membrane is 
glued on the top rigid surface. The bolus is fed into the dialysis membrane via a syringe and pushed manually until the starting position.

The experiment used in the study by Mackley et al. (2013) was improved in several ways: a counterweight to equilibrate the arm weight and an adjustable gap between the roller and the palate were introduced. Furthermore the initial angular position of the roller $\left(\theta_{\mathrm{i}}=45^{\circ}\right)$ is chosen to mimic the contact between the tongue tip and the bolus before an in vivo swallow.

The arm is initially blocked by a pin and different weights can be used to apply a range of torques to the arm. Once the pin is released, the weight pulls the arm and the roller, propelling the bolus inside the membrane. The applied force and bolus physical properties determine the resulting acceleration and the evolution of bolus angular position with time. The roller travels to the final position $\theta_{\mathrm{f}}=165^{\circ}$, as depicted in Figure 1 and the liquid flows in the membrane until it reaches the open end and flows out. Although the epiglottis is schematically represented in the model, it is not considered in this work.

In order to measure quantitatively the arm and liquid motion, this is recorded using a Photron FastCam SA3 high speed camera, at a frame rate of 500 frames/s. Image analysis is used to extract the angular position of the roller (bolus tail), the bolus front and the transit time. Finally, the residual liquid mass in the system is obtained by weighting.

Exploiting the advantages provided by the model swallowing experiment, the process parameters were varied independently to elucidate their effect on the liquid motion. In particular we considered the effect of the liquid viscosity $\mu$, the driving force $\mathrm{F}$, the liquid volume $\mathrm{V}$ and the gap between the roller and the membrane h', see Table 1

The force and the pressure applied by the roller on the bolus increase during an experiment because of the transient motion and the inertia of the system. This will be discussed after introducing the theory. The maximum driving force at the roller $F_{R}$ and the pressure applied on the bolus $\mathrm{P}_{(\mathrm{R} / \text { bolus })}$ can be estimated 
from a torque balance on the system at steady state:

$$
\mathrm{F}_{\mathrm{R}}=\frac{\mathrm{r}_{\mathrm{C}}}{\mathrm{r}_{\mathrm{A}}+\mathrm{r}_{\mathrm{R}}} \mathrm{F}=\frac{\mathrm{r}_{\mathrm{C}}}{\mathrm{r}_{\mathrm{A}}+\mathrm{r}_{\mathrm{R}}} \mathrm{mg}=\mathrm{P}_{(\mathrm{R} / \text { bolus })} \mathrm{S}
$$

110 $\mathrm{mm}) . \mathrm{r}_{\mathrm{C}}$ is the distance from the center of rotation of the point of application of the driving force $(28 \mathrm{~mm})$, while $\mathrm{r}_{\mathrm{A}}(47 \mathrm{~mm})$ is the distance of the center of the roller from the center of rotation. The contact area $\mathrm{S}$ between the roller and the membrane is approximately $1.6 \mathrm{~cm}^{2}$.

In the range of applied driving force $(\mathrm{F}=1-5 \mathrm{~N})$, the maximum pressure is estimated to vary in the range $3.4-17.2 \mathrm{kPa}$, thus covering most of the range measured during in vivo experiments (Yokoyama et al., 2014, Nicosia et al. 2000, Youmans and Stierwalt, 2006; Clavé et al., 2006).

A qualitative comparison of the flow during a model swallow and in vivo imaging is also presented. The latter was obtained using a Siemens Ultrasound SC2000 and a 8v3c curvilinear probe, during the development of the method that conducted to the preclinical phase of the clinical trial with reference $03 / 12$, approved by the Commission Cantonale d'Éthique de la Recherche Sur l'Être Humain, Vaud, Switzerland. Participant written informed consent was collected

\subsection{Theory}

A theory was developed to interpret the flow observed during the artificial swallows and to assess the relative importance of the different phenomena.

We write the conservation of the angular momentum of the system, considering the system angular acceleration $\ddot{\theta}$ and total momentum of inertia, the applied force and the viscous dissipation.

$$
\left(\mathrm{I}_{\text {tot } / \mathrm{O}}+\mathrm{I}_{\text {bolus } / \mathrm{O}}+\frac{\mathrm{F}}{\mathrm{g}} \mathrm{r}_{\mathrm{C}}^{2}\right) \ddot{\theta}=\mathrm{Fr}_{\mathrm{C}}-\mathrm{F}_{\mathrm{v}}\left(\mathrm{r}_{\mathrm{A}}+\mathrm{r}_{\mathrm{R}}\right)
$$




\begin{tabular}{|c|c|c|c|c|c|c|c|c|c|}
\hline Exp. & $\mu(\mathrm{Pa} \mathrm{s})$ & $F(\mathrm{~N})$ & $V(\mathrm{~mL})$ & $h^{\prime}(\mathrm{mm})$ & Exp. & $\mu(\mathrm{Pa} \mathrm{s})$ & $F(\mathrm{~N})$ & $V(\mathrm{~mL})$ & $h^{\prime}(\mathrm{mm})$ \\
\hline a1 & 0.053 & 1 & 6 & 0.2 & $\mathrm{~d} 6$ & 0.053 & 2 & 6 & 1.4 \\
\hline $\mathrm{a} 2$ & 0.053 & 1.3 & 6 & 0.2 & $\mathrm{~d} 7$ & 0.053 & 3 & 6 & 0.1 \\
\hline a3 & 0.053 & 1.5 & 6 & 0.2 & $\mathrm{~d} 8$ & 0.053 & 3 & 6 & 0.2 \\
\hline $\mathrm{a} 4$ & 0.053 & 2 & 6 & 0.2 & d9 & 0.053 & 3 & 6 & 0.4 \\
\hline a5 & 0.053 & 2.7 & 6 & 0.2 & $\mathrm{~d} 10$ & 0.053 & 3 & 6 & 0.6 \\
\hline $\mathrm{a} 6$ & 0.053 & 4 & 6 & 0.2 & $\mathrm{~d} 11$ & 0.053 & 3 & 6 & 0.8 \\
\hline a7 & 0.053 & 5 & 6 & 0.2 & $\mathrm{~d} 12$ & 0.053 & 3 & 6 & 1.4 \\
\hline b1 & 0.006 & 2 & 6 & 0.2 & $\mathrm{~d} 13$ & 0.053 & 4 & 6 & 0.1 \\
\hline $\mathrm{b} 2$ & 0.053 & 2 & 6 & 0.2 & d14 & 0.053 & 4 & 6 & 0.2 \\
\hline b3 & 0.441 & 2 & 6 & 0.2 & $\mathrm{~d} 15$ & 0.053 & 4 & 6 & 0.4 \\
\hline $\mathrm{b} 4$ & 0.788 & 2 & 6 & 0.2 & $\mathrm{~d} 16$ & 0.053 & 4 & 6 & 0.6 \\
\hline b5 & 1.185 & 2 & 6 & 0.2 & $\mathrm{~d} 17$ & 0.053 & 4 & 6 & 0.8 \\
\hline $\mathrm{c} 1$ & 0.006 & 4 & 6 & 0.2 & $\mathrm{~d} 18$ & 0.053 & 4 & 6 & 1.4 \\
\hline $\mathrm{c} 2$ & 0.053 & 4 & 6 & 0.2 & e1 & 0.441 & 4.1 & 2 & 0.1 \\
\hline $\mathrm{c} 3$ & 0.441 & 4 & 6 & 0.2 & $\mathrm{e} 2$ & 0.441 & 4.1 & 2 & 0.2 \\
\hline $\mathrm{c} 4$ & 0.788 & 4 & 6 & 0.2 & e3 & 0.441 & 4.1 & 4 & 0.1 \\
\hline $\mathrm{c} 5$ & 1.185 & 4 & 6 & 0.2 & $\mathrm{e} 4$ & 0.441 & 4.1 & 4 & 0.2 \\
\hline $\mathrm{d} 1$ & 0.053 & 2 & 6 & 0.1 & e5 & 0.441 & 4.1 & 6 & 0.1 \\
\hline $\mathrm{d} 2$ & 0.053 & 2 & 6 & 0.2 & e6 & 0.441 & 4.1 & 6 & 0.2 \\
\hline $\mathrm{d} 3$ & 0.053 & 2 & 6 & 0.4 & e7 & 0.441 & 4.1 & 8 & 0.1 \\
\hline $\mathrm{d} 4$ & 0.053 & 2 & 6 & 0.6 & e8 & 0.441 & 4.1 & 8 & 0.2 \\
\hline $\mathrm{d} 5$ & 0.053 & 2 & 6 & 0.8 & - & - & - & - & - \\
\hline
\end{tabular}

Table 1: Summary of the experiments performed in this study. 
The total inertia of the rotating arm, roller and counterweight $\left(\mathrm{I}_{\text {tot/O }}\right)$ was obtained from the dynamics of the experiments without liquid bolus. The momentum of inertia of the bolus placed in the soft tube $\left(\mathrm{I}_{\text {bolus } / \mathrm{O}}\right)$ was calculated from its mass. The last term of equation 2 accounts for the viscous dissipation :

$$
\mathrm{F}_{\mathrm{v}}=\beta 8 \pi \operatorname{Lr}_{\mathrm{A}} \mu \dot{\theta}
$$

where $\mathrm{L}$ is the bolus length. Since the latter was observed to be almost constant during all experiments, the viscous dissipation can be easily derived from the roller velocity. $\beta$ is an empirical correction of the Poiseuille expression that accounts for the non-circular section of the membrane and the squeezing action of the roller. Considering $\beta=2$ was found to guarantee a good agreement between theory and experiments. This value corresponds to the flow in an elliptical section with an aspect ratio of five and was kept constant throughout this study.

Experimentally, we have also observed the existence of a threshold in driving force, $F_{\min }$ that is required to propel the bolus through the flexible tube and depends on the bolus properties. Consequently the driving force $\mathrm{F}$ is substituted by $\mathrm{F}-\mathrm{F}_{\min }$.

After integration, the angular velocity of the roller (and bolus tail) reads :

$$
\dot{\theta}(\mathrm{t})=\frac{\mathrm{r}_{\mathrm{C}}\left(\mathrm{F}-\mathrm{F}_{\min }\right)}{8 \pi \mathrm{Lr}_{\mathrm{A}}\left(\mathrm{r}_{\mathrm{A}}+\mathrm{r}_{\mathrm{R}}\right) \beta \mu}\left(1-\exp \left(\frac{-8 \pi \mathrm{Lr}_{\mathrm{A}}\left(\mathrm{r}_{\mathrm{A}}+\mathrm{r}_{\mathrm{R}}\right) \beta \mu \mathrm{t}}{\mathrm{I}_{\text {tot } / \mathrm{O}}+\mathrm{I}_{\text {bolus } / \mathrm{O}}+\frac{\mathrm{Fr}_{\mathrm{C}}{ }^{2}}{\mathrm{~g}}}\right)\right)
$$

\section{Results}

Figure 2 compares the in vivo swallow of $10 \mathrm{~mL}$ of sugar molasses $(\mu=0.850$ Pa s) with an artificial swallow of $6 \mathrm{~mL}$ of an aqueous glycerol solution of similar viscosity $(\mu=0.79 \mathrm{~Pa} \mathrm{~s}$ ). In the model experiments the bolus volume was adapted to maintain comparable bolus length and height and the driving force was set to $\mathrm{F}=1.4 \mathrm{~N}$ which corresponds approximately to $\mathrm{P}=4.8 \mathrm{kPa}$ (see Section Materials and Methods), consistently with tongue pressures reported 
in the literature (Yokoyama et al. 2014, Nicosia et al., 2000; Youmans and Stierwalt, 2006: Clavé et al., 2006). The movement of the roller induces a normal displacement of the soft membrane holding the liquid, toward the rigid top wall, similarly to the tongue approaching the palate. The geometrical, kinematic and dynamic agreement of the bolus flow can be qualitatively appreciated in Figure 2. A more detailed comparison will be the object of another publication (Mowlavi et al., 2015) .

The influence of the driving force on the flow was ascertained using a liquid viscosity $\mu=0.053 \mathrm{~Pa} \mathrm{~s}$ and various applied forces. Figure 3 shows several typical snapshots illustrating the position of the liquid at different times in a given experiment (along a row) and the effect of varying the applied forces (along a column). In particular, the snapshots taken at $0.3 \mathrm{~s}$ show clearly the effect of the applied force. At this time, the bolus has already left the membrane when the highest force is applied, but not in the other cases.

The bolus length was found to remain constant during the experiments, until the bolus head left the tubing. Therefore, only the position and the velocity of the roller (i.e. the bolus tail) was considered. Figure 4 a shows the typical evolution of the angular position of the roller (i.e. of the bolus tail) and the good reproducibility. The experimental angular velocities are plotted using solid lines in Figure $4 \mathrm{~b}$, up to when the bolus front reaches the end of the soft tube. Higher driving forces reduce the liquid transit time. As already anticipated, it was observed that below a threshold driving force the bolus is not propelled through the tube. The theoretical predictions are plotted using dashed lines and agree well with the experimental results (solid lines).

Figure $5 \mathrm{a}$ and $5 \mathrm{~b}$ show the effect of the bolus viscosity on the angular velocity evolution for $\mathrm{F}=2 \mathrm{~N}$ and $\mathrm{F}=4 \mathrm{~N}$, respectively. An increase in viscosity leads to a decrease in acceleration of the bolus tail and to an increase of the transit time. The theoretical predictions are plotted using dashed lines and agree well with experiments. Similar results were obtained when varying bolus volume 
and the width of the gap between the roller and the rigid surface mimicking the palate. At constant applied force, the bolus motion is slowed down by an increased bolus volume or a smaller gap.

Figure 6 summarizes the dependence of the residual mass of liquid on viscosacceleration. During the viscous regime at constant angular velocity, the force 
and pressure exerted by the roller is very close to the maximum values introduced at the end of paragraph Methods 2.2. For the lowest viscosity liquids, only the constant acceleration regime appears. Figure $5 \mathrm{~b}$ shows that higher driving forces, namely $F=4 N$, induce a constant acceleration regime for most of the experiment duration, itself shortened with respect to lower forces. In all cases, the theoretical results (dashed lines) agree well with the experimental results (solid lines).

Figure 7 illustrates the use of our theory to predict the combined effect of liquid viscosity and of the net driving force. At low liquid viscosity, the transient regime at constant acceleration dominates the process and the (Newtonian) viscosity has little influence on its dynamics, whereas a much stronger effect and slower dynamics are observed if the steady viscous regime is reached, namely for highly viscous liquids. Conversely, liquid density can only have some effect on the swallowing time when the constant acceleration regime holds for most of the swallow. Nicosia and Robbins (2001) claimed in their study that density dominates the flow in the oral phase when liquid viscosity is lower than $0.1 \mathrm{Pas}$. The system presented in this study simulates much more closely the human physiology because it considers a spatially variable gap between the tongue and the palate that can transport the bolus into the oesophagous (Figure 2). However, this conclusion still holds, as shown by the flat isochronic curves in Figure 7, when viscosity is lower than $0.1 \mathrm{~Pa}$ s. Finally, the theoretical contour lines in Figure 7 suggest to which extent the applied force for swallowing should 235 increase to counterbalance an increase in viscosity, while maintaining a constant transit time.

A lower swallowing force has been shown to induce a decrease in bolus acceleration and velocity and an increase in the oral transit time. Clavé et al. (2006) observed the same effect in patients affected by brain damage or neurodegenerative conditions. Naturally, other perturbations of the swallowing process can be linked to the same conditions and our model experiments do not currently have 
the ambition of replicating any specific condition, but rather to verify simple mechanical links between the well controlled parameters of this model system and the resulting liquid flow.

Our theory and experiments can also help formulating a hypothesis concerning the higher pressure measured at the back of the tongue, during swallowing, by Nicosia et al. (2000). The tongue and the bolus are initially static and because of the initial acceleration and the tongue inertia, the force exerted by the roller on the liquid and its pressure increase over time. Therefore, the higher 250 pressure measured at the back of the tongue might be simply caused by the lower acceleration of the tongue and the bolus, when the latter transits throughout the back of the tongue.

A bolus residue after a swallow is generally not desired because it can potentially lead to aspiration after the airway protection is released. Increasing the gap between the roller and the palate leads to an increase in the bolus residual mass as shown in Figure 6. This effect could potentially explain the condition of some elderly patients with poor tongue mobility, resulting in incomplete sealing. The high residual mass obtained with high gap explains also partly the lower observed transit time, because the volume of the bolus being transported in the tube decreases significantly during the swallow.

For Newtonian liquids, the effect of the viscosity on residues is not very strong, as shown in Figure 6, with the exception of the highest viscosity tested that generates more residues. Two mechanisms were considered to explain the increase of residues at high liquid viscosity. On one side the roller might slightly deform at high liquid viscosity, thus increasing the effective gap and the resulting residual film. This hypothesis could be discarded by considering that the roller elastic modulus is approximately $2500 \mathrm{MPa}$ and the maximum pressure $17 \mathrm{kPa}$, leading to a maximum strain below $10^{-5}$. The maximum deformation of the roller is therefore always negligible with respect to the gap. Nevertheless, the effect of using rollers with an increased compliance and/or roughness certainly 
deserves to be studied in the future. Another mechanism that could explain the residue increase when high viscosity liquids are swallowed, is a reduced effectiveness of the peristaltic flow. Brown and Hung (1977) considered the steady viscous flow induced by a peristaltic wave in a bi-dimensional channel. When the wave amplitude is much smaller than the wavelength, the transported flow rate decreases when the liquid viscosity increases, which would be consistent to observing a residue increase in our configuration. Brown and Hung (1977) claimed also that this trend reverses for finite amplitude to wavelength ratios, which could explain the existence of a range of viscosities having little or no impact on residues. Further experiments with different roller diameters would be necessary to support this interpretation.

The cause of this increase in residue mass notwithstanding, the results obtained in this model experiment support the observations made in a recent review (Steele et al., 2014) of in vivo results: increasing bolus viscosity has the positive effect of slowing down the flow, allowing more time for preparing the airway closure. However, a too high viscosity can induce higher residues.

Finally, our experimental results suggest also a weak dependence of oral residues on bolus volume. Splitting a bolus in several smaller volumes could therefore increase the cumulated residual mass. However, it should be underlined that this conclusion considers only the residues in the oral phase and not the likelihood of aspiration during swallowing, nor pharyngeal residues, since the flow in the pharynx is not accounted for in this study.

Beside the already mentioned geometrical simplification of the oral cavity, several other assumptions would deserve being refined in future studies. Given the fast dynamics, this study assumes that no active control takes place during swallowing and that the process takes place under the action of an imposed force. A more detailed comparison of the dynamics of model experiments and in vivo flow will be the focus of another publication (Mowlavi et al., 2015). Several other extensions of this study can be envisaged to pave the way toward 
cesses involving the pharynx and the epiglottis, the risk of aspiration during swallowing, the flow of non-Newtonian liquids or saliva lubrication.

\section{Conclusions and perspectives}

We have developed a quantitative model experiment that helps understanding the oral phase of swallowing, based on a qualitative prototype originally designed by Mackley et al. (2013).

We have compared this experiment with in vivo ultrasound observations and used it to study quantitatively the changes in transit time induced by varying the applied force and/or the viscosity of a Newtonian liquid. We have established a realistic theory that highlights the existence of two different regimes: an initial constant acceleration regime, possibly followed by a regime at constant velocity, dominated by the viscous dissipation. The liquid viscosity has, conversely, no effect on the initial regime. A poor tongue-palate sealing (i.e. a higher gap) and a very high liquid viscosity lead to a higher liquid residue, while the latter does not depend on the liquid volume. This study provides a mechanistic explanation to some in vivo observations from other studies, for instance the fact that increasing too much the liquid viscosity can induce higher residues, beside slowing down the swallowing process enough to obtain an effective airway closure. Furthermore, the theory shows that inertia can explain why during swallowing the liquid pressure is higher at the back of the tongue.

This model experiment and theoretical considerations are the first step toward a mechanical understanding of human swallowing, that can contribute to foster the development of improved dysphagia treatments. Interesting extensions of this study can be envisaged: considering shear thinning liquid boluses or integrating other physiological processes (pharynx, epiglottis, saliva), in order to consider the risk of aspiration stemming from a reduced coordination. 


\section{Acknowledgments}

This study was funded by Nestlé Research. The authors thank Prof. M. Mackley and Dr. S .Butler for providing access to the artificial throat prototype developed at U.Cambridge, NRC workshop for designing and manufacturing the model swallowing experiment used in this study and Mrs. B. Aubert for the technical assistance with the ultrasound measurements.

\section{Conflict of interest statement}

The authors have no conflicts of interest to declare.

335

Adnerhill, I., Ekberg, O., Groher, M.E., 1989. Determining normal bolus size for thin liquids. Dysphagia 4, 1-3.

Brown, T., Hung, T.K., 1977. Computational and experimental investigations of two-dimensional nonlinear peristaltic flows. J. Fluid Mechanics 83, 249-272.

Clavé, P., De Kraa, M., Arreola, V., Girvent, M., Farré, R., Palomera, E., Serra-Prat, M., 2006. The effect of bolus viscosity on swallowing function in neurogenic dysphagia. Alimentary Pharmacology and Therapeutics 24, $1385-1394$.

De Loubens, C., Magnin, A., Doyennette, M., Tréléa, I.C., Souchon, I., 2011. A biomechanical model of swallowing for understanding the influence of saliva and food bolus viscosity on flavor release. Journal of Theoretical Biology 280, $180-188$.

De Loubens, C., Magnin, A., Verin, E., Doyennette, M., Tréléa, I.C., Souchon, I., 2010. A lubrication analysis of pharyngeal peristalsis: Application to 350 flavour release. Journal of Theoretical Biology 267, 300 - 311. 
Dodds, W., 1989. The physiology of swallowing. Dysphagia 3, 171-178.

Ho, A.K., Tsou, L., Green, S., Fels, S., 2014. A 3d swallowing simulation using smoothed particle hydrodynamics. Computer Methods in Biomechanics and Biomedical Engineering: Imaging \& Visualization 2, 237-244.

Kikuchi, T., Michiwaki, Y., Kamiya, T., Toyama, Y., Tamai, T., Koshizuka, S., 2015. Human swallowing simulation based on videofluorography images using hamiltonian mps method. Computational Particle Mechanics , 1-14.

Mackley, M., Tock, C., Anthony, R., Butler, S., Chapman, G., Vadillo, D., 2013. The rheology and processing behavior of starch and gum-based dysphagia thickeners. Journal of Rheology 57, 1533-1553.

Mowlavi, S., Engmann, J., Burbidge, A., Le Reverend, B., Hayoun, P., Ramaioli, M., 2015. In vivo observations and model experiments on the oral phase of swallowing of newtonian and shear thinning liquids. in prep. .

Nicosia, M., Hind, J., Roecker, E., Carnes, M., Doyle, J., Dengel, G., Robbins, J., 2000. Age effects on the temporal evolution of isometric and swallowing pressure. Journals of Gerontology - Series A Biological Sciences and Medical Sciences 55, M634-M640.

Nicosia, M.A., Robbins, J., 2001. The fluid mechanics of bolus ejection from the oral cavity. Journal of Biomechanics 34, 1537-1544. J.A.Y., Coutts, K., Dantas, R.O., Duivestein, J., Giosa, L., Hanson, B., Lam, P., Lecko, C., Leigh, C., Nagy, A., Namasivayam, A.M., Nascimento, W.V., Odendaal, I., Smith, C.H., Wang, H., 2014. The influence of food texture and liquid consistency modification on swallowing physiology and function: 375 A systematic review. Dysphagia 30, 2-26. 
Utanohara, Y., Hayashi, R., Yoshikawa, M., Yoshida, M., Tsuga, K., Akagawa, Y., 2008. Standard values of maximum tongue pressure taken using newly developed disposable tongue pressure measurement device. Dysphagia 23, 286-290.

380 Woda, A., Mishellany-Dutour, A., Batier, L., Franois, O., Meunier, J.P., Reynaud, B., Alric, M., Peyron, M.A., 2010. Development and validation of a mastication simulator. Journal of Biomechanics 43, 1667-1673.

Yokoyama, S., Hori, K., Tamine, K.I., Fujiwara, S., Inoue, M., Maeda, Y., Funami, T., Ishihara, S., Ono, T., 2014. Tongue pressure modulation for initial gel consistency in a different oral strategy. PLoS ONE 9.

Youmans, S.R., Stierwalt, J.A., 2006. Measures of tongue function related to normal swallowing. Dysphagia 21, 102-111. 

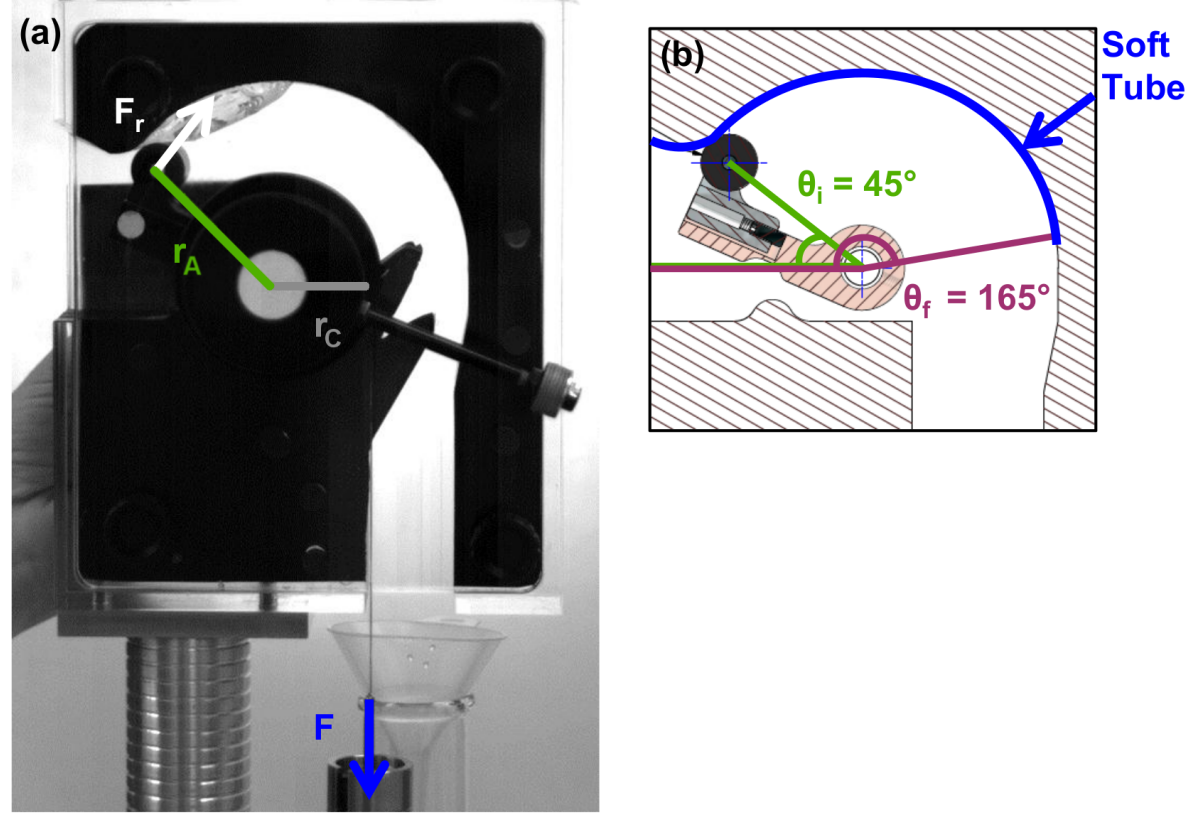

Figure 1: Artificial swallowing experiment : (a) overview of the experimental setup, (b) detailed drawing of the arm and roller without liquid. 


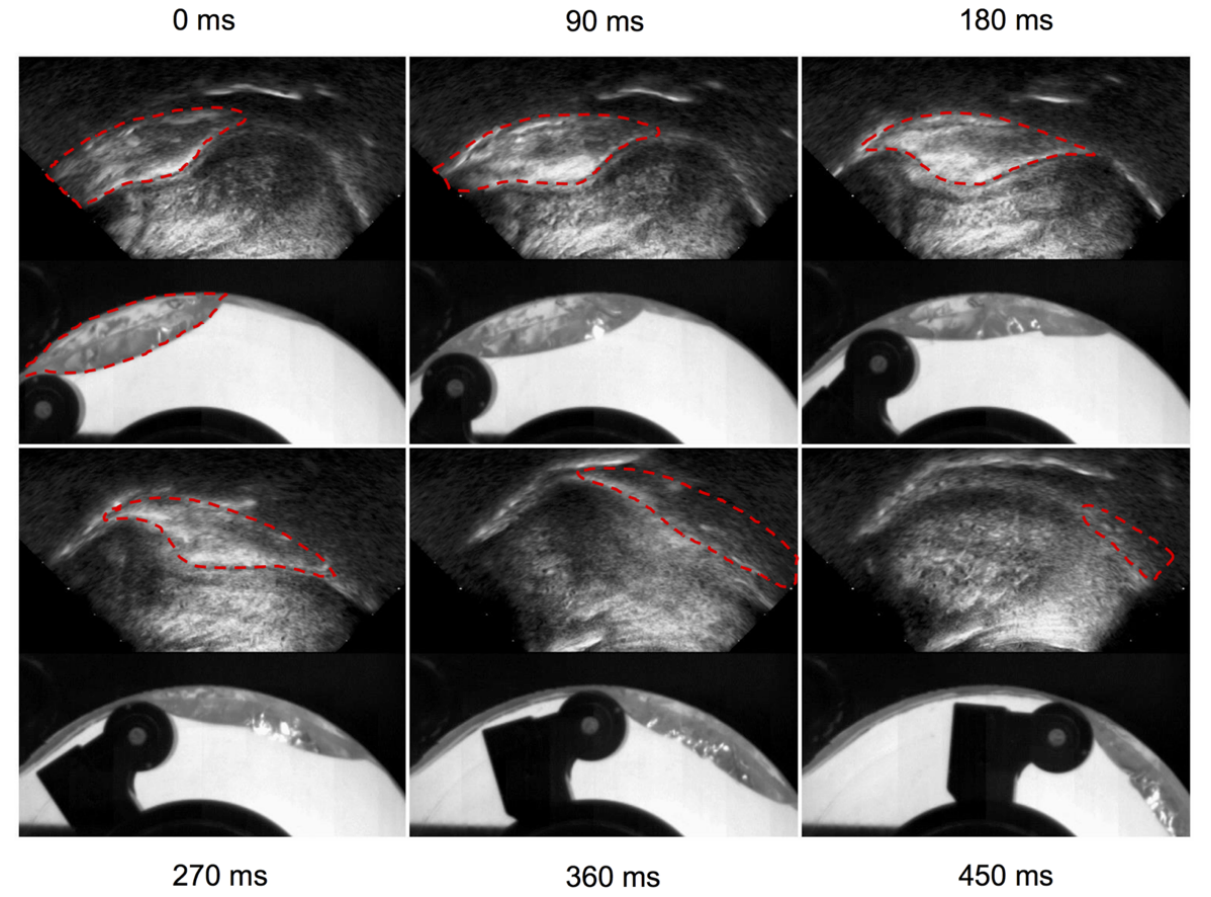

Figure 2: Comparison between an in vivo swallow of sugar molasses (top) and an artificial swallow of an aqueous glycerol solution of similar viscosity (bottom). Images are taken from the side. In vivo, the liquid is highlighted in red and is hold by the tongue that appears darker where contracted. 


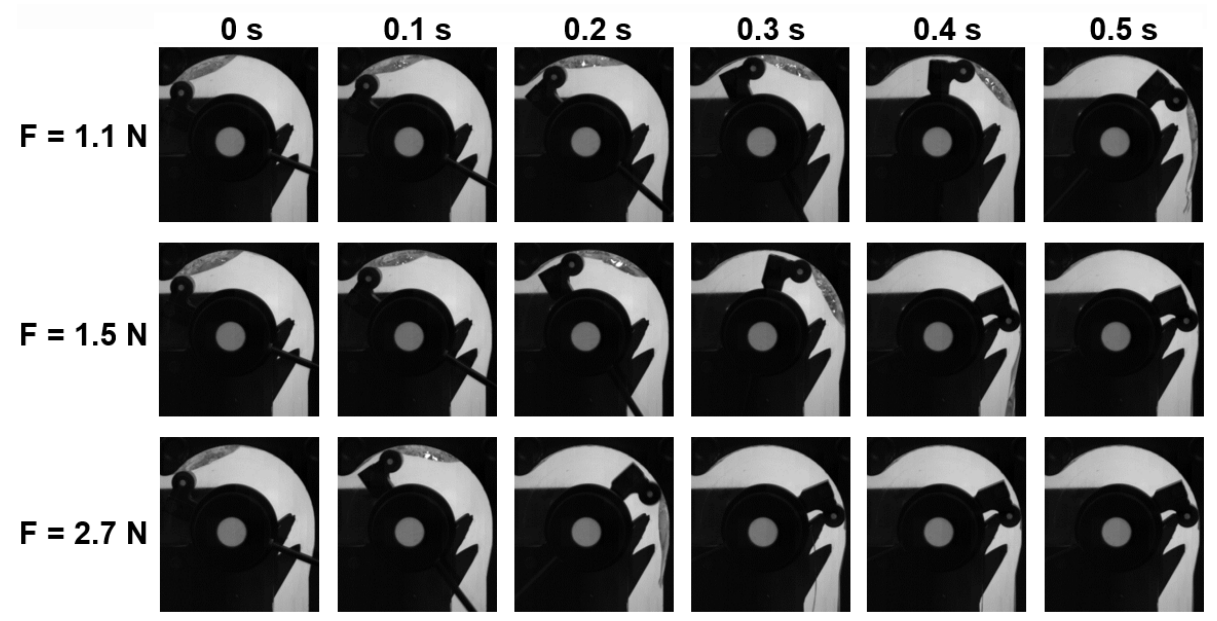

Figure 3: Several snapshots illustrating the liquid flow in the model swallowing experiment, using a bolus viscosity $\mu=0.053 \mathrm{~Pa}$ s. Snapshots from different experiments using different applied forces (1.1 to $2.7 \mathrm{~N}$ ) are reported in different rows. 

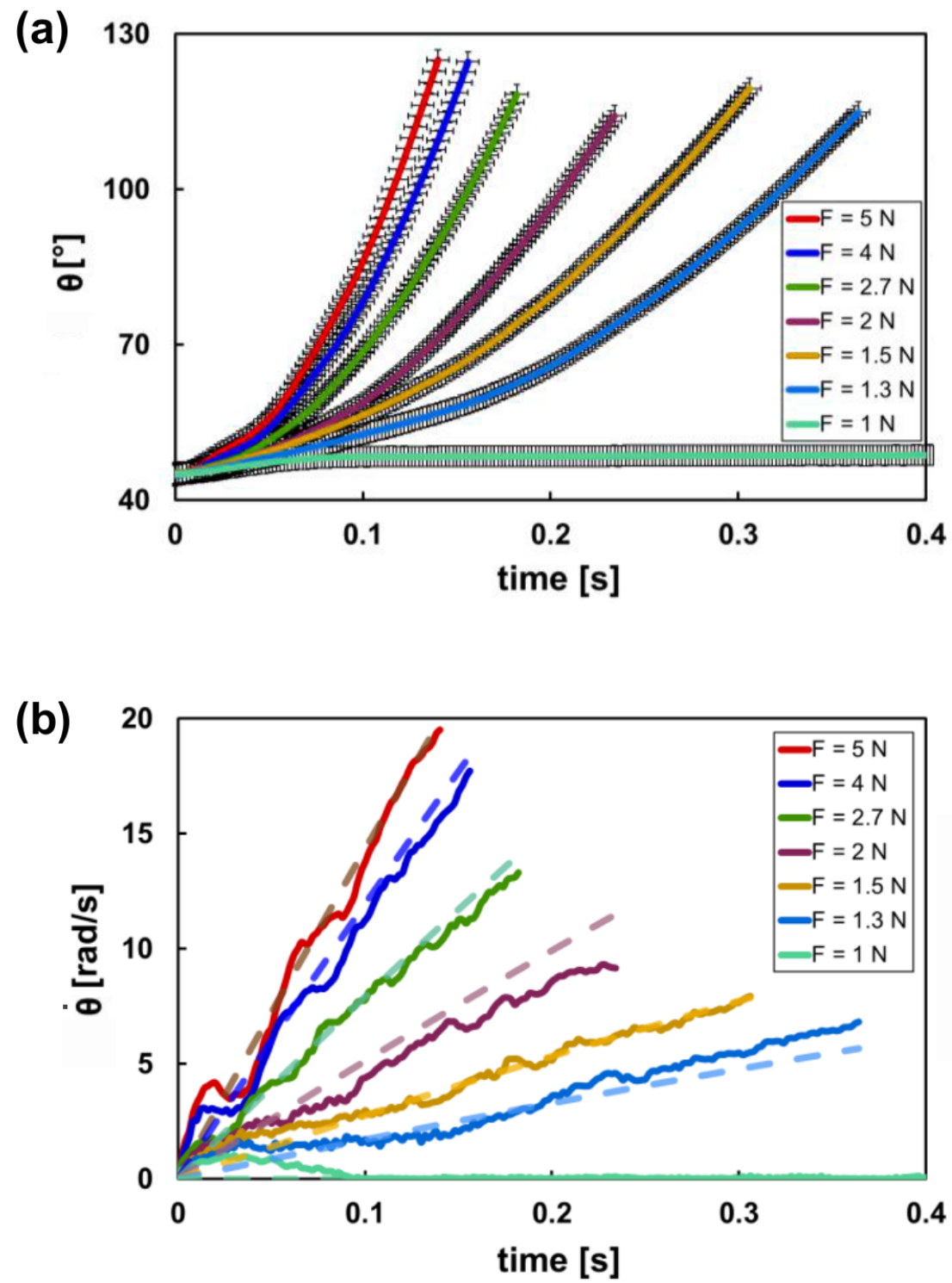

Figure 4: Effect of the driving force on the flow of a $\mu=0.053 \mathrm{~Pa}$ s liquid, using $h^{\prime}=0.2 \mathrm{~mm}$, $V_{0}=6 \mathrm{~mL}-(\mathrm{a})$ angular position of bolus tail (b) angular velocity of bolus tail. In the latter, experimental results are plotted using solid lines and theoretical results using dashed lines. 

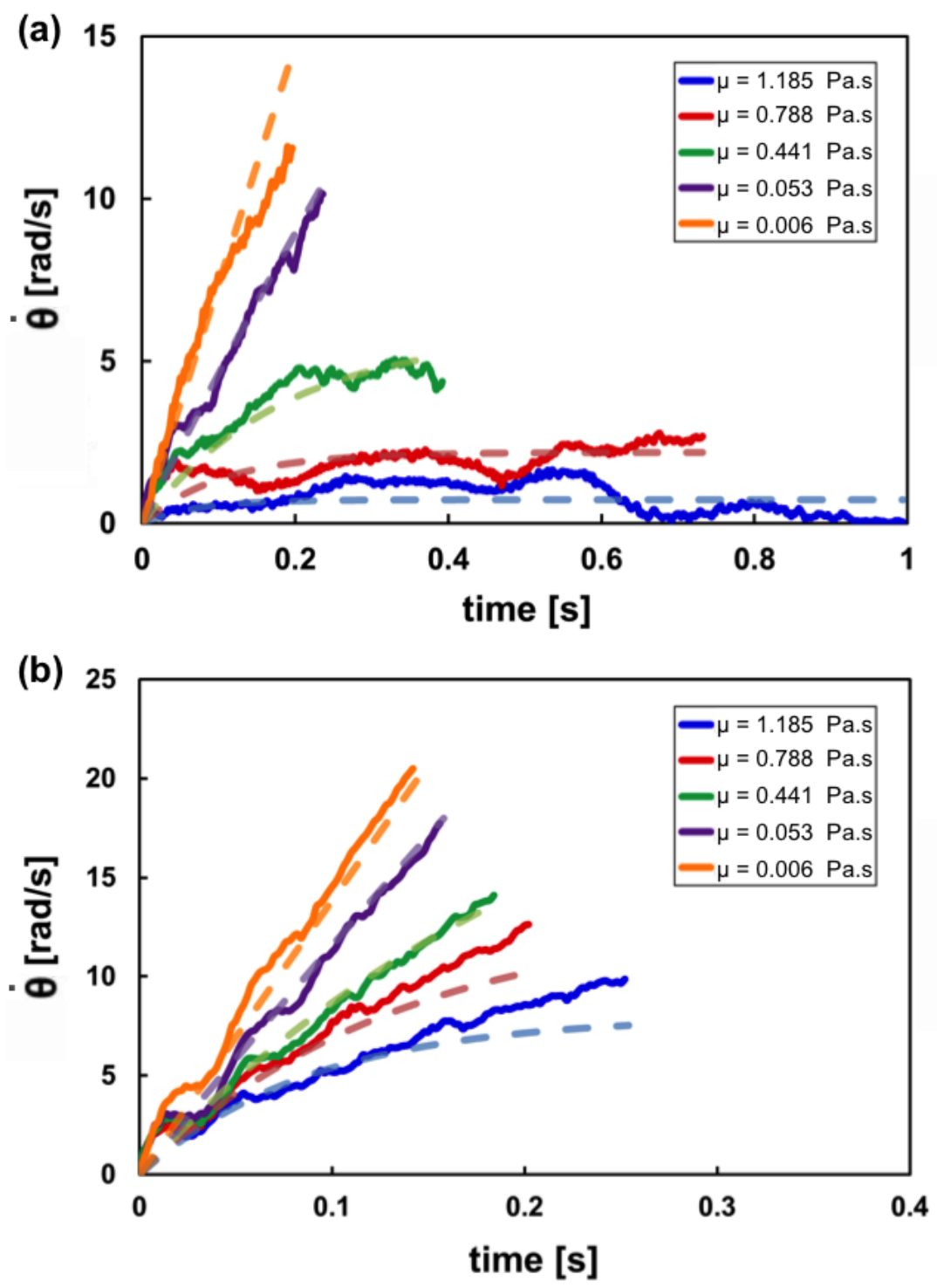

Figure 5: Effect of liquid viscosity on the angular velocity of the bolus tail for a driving force of $F=2 \mathrm{~N}$ (a) and $F=4 \mathrm{~N}$ (b). Experimental results are plotted using solid lines and theoretical results using dashed lines. 

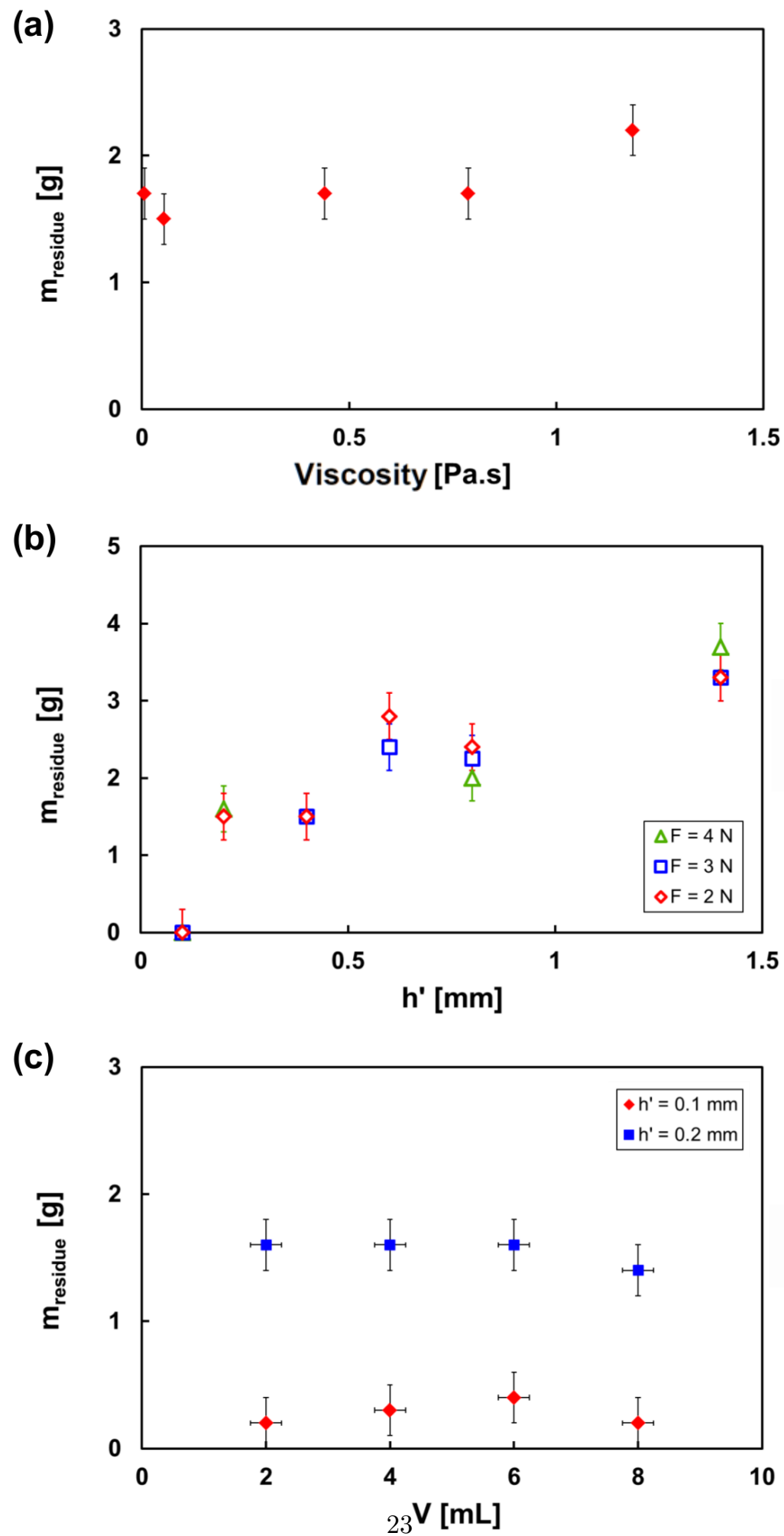

Figure 6: Residual bolus mass as a function of (a) the bolus viscosity for $F=4 \mathrm{~N}, h^{\prime}=0.2$ $\mathrm{mm}, V_{0}=6 \mathrm{~mL}$ - (b) the gap and the driving force, $\mu=0.053 \mathrm{~Pa} \mathrm{~s}, V_{0}=6 \mathrm{~mL}-(\mathrm{c})$ the bolus volume and the gap, $F=4.1 \mathrm{~N}, \mu=0.441 \mathrm{~Pa} \mathrm{~s}$ 


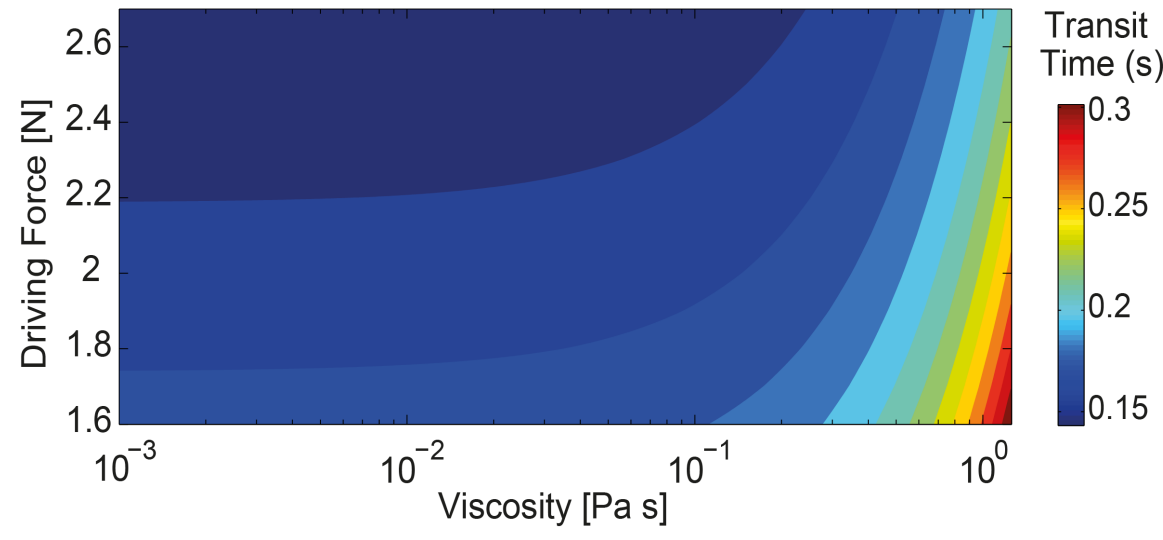

Figure 7: Influence of the net applied force and of the liquid viscosity on the bolus transit time predicted by the theory. 